\title{
Correction to: Automatic Segmentation of Vestibular Schwannoma from T2-Weighted MRI by Deep Spatial Attention with Hardness-Weighted Loss
}

Guotai Wang, Jonathan Shapey, Wenqi Li, Reuben Dorent, Alexis Dimitriadis, Sotirios Bisdas, Ian Paddick, Robert Bradford,

Shaoting Zhang, Sébastien Ourselin, and Tom Vercauteren

Correction to:

Chapter "Automatic Segmentation of Vestibular Schwannoma from T2-Weighted MRI by Deep Spatial Attention with Hardness-Weighted Loss" in: D. Shen et al. (Eds.):

Medical Image Computing and Computer Assisted

Intervention - MICCAI 2019, LNCS 11765,

https://doi.org/10.1007/978-3-030-32245-8_30

The original version of this chapter was revised. An author's name was misspelled. The name has been corrected to Alexis Dimitriadis. 